\title{
Neurotropism of SARS-CoV-2 and neurological diseases of the central nervous system in COVID-19 patients
}

\author{
Shobi Veleri ${ }^{1}$ \\ Received: 8 July 2021 / Accepted: 8 October 2021 / Published online: 25 October 2021 \\ (c) The Author(s), under exclusive licence to Springer-Verlag GmbH Germany, part of Springer Nature 2021
}

\begin{abstract}
The devastating COVID-19 pandemic is caused by the SARS-CoV-2 virus. It primarily affects the lung and induces acute respiratory distress leading to a decrease in oxygen supply to the cells. This lung insufficiency caused by SARS-CoV-2 virus contributes to hypoxia which can affect the brain and other organ systems. The heightened cytokine storm in COVID-19 patients leads to an immune reaction in the vascular endothelial cells that compromise the host defenses against the SARS$\mathrm{CoV}-2$ virus in various organs. The vascular endothelial cell membrane breach allows access for SARS-CoV-2 to infect multiple tissues and organs. The neurotropism of spike protein in SARS-CoV-2 rendered by furin site insertion may increase neuronal infections. These could result in encephalitis and encephalopathy. The COVID-19 patients suffered severe lung deficiency often showed effects in the brain and neural system. The early symptoms include headache, loss of smell, mental confusion, psychiatric disorders and strokes, and rarely encephalitis, which indicated the vulnerability of the nervous system to SARS-CoV-2. Infection of the brain and peripheral nervous system can lead to the dysfunction of other organs and result in multi-organ failure. This review focuses on discussing the vulnerability of the nervous system based on the pattern of expression of the receptors for the SARS-CoV-2 and the mechanisms of its cell invasion. The SARS-CoV-2 elicited immune response and host immune response evasion are further discussed. Then the effects on the nervous system and its consequences on neuro-sensory functions are discussed. Finally, the emerging information on the overall genetic susceptibility seen in COVID-19 patients and its implications for therapy outlook is discussed.
\end{abstract}

Keywords SARS-CoV-2 $\cdot$ Furin $\cdot$ Neurotropism $\cdot$ Encephalopathy $\cdot$ Neural diseases

\section{Introduction}

The year 2019 witnessed the emergence of a new Severe Acute Respiratory Syndrome (SARS) virus causing the incidence of pneumonia in Wuhan. The pneumonia was conspicuous because it was not responding to the standard treatment regimen (Chen et al. 2020a). Its clinical symptoms are fever, dry cough, sore throat, pneumonia, severe breathing difficulty (dyspnoea) and myalgia (Zhou et al. 2020a). The dyspnoea resembled the severe acute respiratory syndrome

Communicated by Sreedharan Sajikumar.

Shobi Veleri

shobi.veleri@icmr.gov.in

1 Drug Safety Division, Indian Council of Medical ResearchNational Institute of Nutrition, Department of Health Research, Ministry of Health and Family Welfare, Government of India, Hyderabad, Telangana 500007, India seen in the SARS caused by a $\beta$-family of coronaviruses, corona virus-1 (SARS-CoV-1), in the pandemic of 2003 (Wan et al. 2020). Therefore, a similar causative agent was suspected, and quickly a new member of $\beta$-family of coronavirus, termed corona virus-2 (SARS-CoV-2) was discovered as the causative agent (Zhou et al. 2020a). Later, the World Health Organization named the disease as Corona Virus Disease of 2019 (COVID-19). SARS-CoV-2 virus shares sequence homology at the genome level with other pathogenic $\beta$-corona viruses like SARS-CoV, Middle East Respiratory Syndrome coronavirus (MERS-CoV), and human coronavirus OC43 (HCoV-OC43). At the whole-genome level, SARS-CoV-2 has the highest nucleotide identity of 79.7\% with SARS-CoV (Zhou et al. 2020b), followed by $54.1 \%$ with MERS-CoV and 51.08\% with HCoV-OC43. At the protein level, the sequence identity for Open Reading Frame-1ab (ORF1ab) of SARS-CoV-2 is $86.51 \%, 48 \%$, $44.93 \%$, respectively, to SARS-CoV, MERS-CoV, and HCoV-OC43. SARS-CoV-2 envelope and nucleocapsid 
proteins have sequence homology of $96 \%$ and $89.6 \%$ with SARS-CoV, respectively. The spike protein of SARS-CoV-2 has a sequence identity homology of $77.62 \%, 31.93 \%$, and $30.84 \%$ with SARS-CoV, MERS-COV, and HCoV-OC43, respectively.

Since January 2020, COVID-19 has emerged as a pandemic and its impact is huge in terms of healthcare burden, economic distress, and human suffering across the globe. The pandemic has been disrupting normal life across all walks of life. As of October 11, 2021, 240 million cases of COVID-19 were recorded with 4.9 million deaths worldwide (WHO 2021) (https://covid19.who.int/). The percentage of people killed was $~ 2 \%$ in closed cases (which had an outcome). Age and sex were vital factors that affected the closed case outcomes. An almost equal proportion of males and females were confirmed with COVID-19. However, males required three times (odds, $\mathrm{OR}=2.84$ ) more intensive care treatment and showed a higher number of deaths (odds, $\mathrm{OR}=1.39$ ) than females. However, age and sex were not a susceptibility factor for the 13 loci associated with COVID-19 in a genome-wide association study (GWAS) (Initiative 2021). The low mortality rate of COVID-19 is the only optimistic outlook the healthcare fraternity initially had when faced with a new virus without a viable therapy. Meanwhile, few new vaccines were developed to guard against the incidence of new infections. These vaccines rendered relative protection against the infection and were capable of reducing the severity of the respiratory symptoms. However, breakthrough infections were often reported in vaccinated people (Hacisuleyman et al. 2021). Besides, a much more severe second wave of COVID-19 globally emerged and it raked America, Europe, India, South America, etc. (Mallapaty 2021; Ferrante et al. 2020; James et al. 2021). Given the enormity of the population infected worldwide, even the $2 \%$ (i.e. $~ 4.9$ million) death rate is alarming.

An enduring issue of COVID-19 patients nagging the healthcare sector is the serious secondary effects other than respiratory distress. The propensity of SARS-CoV-2 to infect the nervous system is highly debated based on various reports of many patients presented with neurological symptoms (Ellul et al. 2020; Maury et al. 2021; Iadecola et al. 2020; Conde Cardona 2020; Meinhardt et al. 2021). Early on in the pandemic, a rare case was reported with only meningoencephalitis even without respiratory distress syndrome (Duong et al. 2020) indicating the central nervous system (CNS) susceptibility for this virus. This review will discuss the CNS vulnerability for SARS-CoV-2 infection, plausible routes of viral entry and the mechanism of viral invasion into the neurons and general mechanisms in cells to defend against the viral infections, and how SARS-CoV-2 evades such inbuilt defense by immune suppression. Then the short-term effects of neural dysfunctions will be discussed. Finally, the genetic susceptibility for COVID-19 and its implication for therapeutic outlook, the possible longterm effects of COVID-19 on patients, and its impact on the healthcare system are discussed.

\section{Molecular mechanism of SARS-CoV-2 and Spike protein's affinity for neurotropism towards Angiotensin-Converting Enzyme-2 (ACE2) receptor}

Coronaviruses are single strand (+) RNA viruses in the order Nidovirales. They normally infect epithelial cells in the respiratory tract, gastrointestinal tract, and kidney. Some of them like the mouse hepatitis virus John Howard Muller strain infected neural cells like oligodendrocytes, astrocytes, and microglia, and developed severe encephalomyelitis. $\mathrm{HCoV-OC43} \mathrm{is} \mathrm{known} \mathrm{to} \mathrm{infect} \mathrm{the} \mathrm{CNS} \mathrm{via} \mathrm{the} \mathrm{nasal} \mathrm{route}$ (Dube et al. 2018). The $\beta$-coronavirus, like its other family members, has four structural proteins: spike (S) protein, a glycoprotein, an envelope (E) protein, membrane (M) glycoprotein, nucleocapsid (N) protein (Fig. 1). The $\mathrm{S}$ protein is a Class I fusion protein present on the viral envelope (Bosch et al. 2003). It mediates the viral attachment to the host cell membrane. The $\mathrm{S}$ protein binds to the ACE2 receptor on the host cell that permits the virus entry into the cell (Fig. 1a). The spike can also utilize other receptors like Basigin (BSG; CD147) and Neuropilin-1 (NRP1) (Wang et al. 2020a; Cantuti-Castelvetri et al. 2020). The $\mathrm{S}$ protein upon binding to the ACE2 receptor is cleaved by protease transmembrane serine protease 2 (TMPRSS2) (Hoffmann et al. 2020). It cleaves the $\mathrm{S}$ into two polypeptides S1 and S2. S1 hosts the receptor-binding domain that docks with the ACE2 receptor. The $\mathbf{S} 2$ forms the stalk of the spike proximal to the viral envelope and it helps the fusion of membranes of virus and host cell. In some coronaviruses, an additional furin-S2' cleavage site is seen distal to the fusion peptide (FP) in S2, which is cleaved by furin. S2' site cleaving enhances the viral entry into the host cell (Shang et al. 2020). Besides TMPRSS- 2 and furin, other proteases like TMPRSS11A may also catalyze virus entry into the cells. TMPRSS11A is expressed in airway epithelial cells and it activates the $\mathrm{S}$ protein of SARS-CoV, MERS, and SARS-CoV-2 (Zhang et al. 2020). It is activated by intracellular autocatalysis. It can cleave S protein at S1/S2 and S2' sites like the TMPRSS-2. The lysosomal protease cathepsin L may also help cell entry of virus given that its inhibitor blocked the entry of SARS-CoV-2 pseudovirus into 293/hACE2 cells. Of note, related cathepsin $\mathrm{B}$ inhibitor did not affect the viral entry (Ou et al. 2020). Similar to SARS-CoV pseudovirus, SARSCoV-2 uses TMPRSS2 and cathepsin, along with furin for cell entry. It seems the spike protein pre-activation by furin renders less dependence on other host cell proteases for cell entry. These receptors and proteases were detected in the 

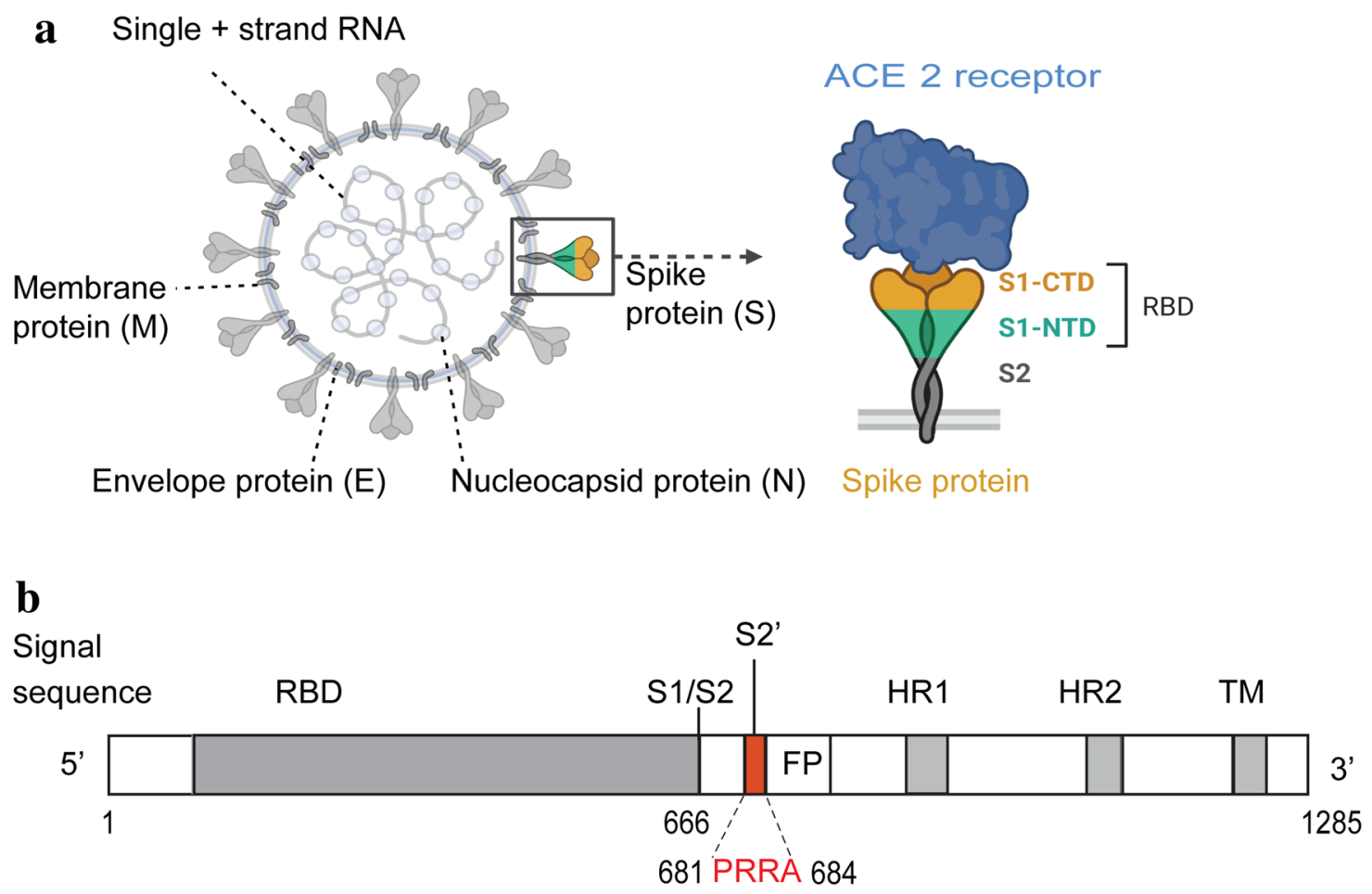

furin amino acids sequence

Fig. 1 Schematic of the SARS-CoV-2 virus, Spike protein and ACE2 receptor with furin site. a The SARS-CoV-2 structure of the virus showing single (plus) strand RNA with its envelope (E), membrane (M) protein, nucleocapsid $(\mathrm{N})$, and spike $(\mathrm{S})$ protein. The $\mathrm{S}$ bound with Angiotensin-Converting Enzyme -2 (ACE2) is depicted. RBD is the receptor-binding domain, S1-CTD and S1-NTD are C-terminal and N-terminal domains of S1. S2 is the C-terminal part of the

susceptible tissues including in the nerve tissues (Iadecola et al. 2020; Chen 2020), though the gene expression levels varied across the tissues analyzed. SARS-CoV-2's plausible neurotropism was noted during the pandemic (Conde Cardona 2020). The furin cleaving site in the spike protein of some coronaviruses rendered them neurotropism (Cheng et al. 2019). A similar furin site is also present in SARS$\mathrm{CoV}-2$ and it has four amino acids proline $(\mathrm{P})$, arginine $(\mathrm{R})$, alanine(A)- PRRA (Fig. 1b) (Andersen et al. 2020). This similarity predicts a propensity for neurotropism. SARSCoV-2 has a higher binding affinity with ACE2 receptors than other $\beta$-coronaviruses due to the slightly higher positive charge in $\mathrm{S}$ protein, which can promote higher virulence (Hassanzadeh et al. 2020). SARS-CoV-2 S protein has four positively charged amino acids more, and five negatively charged amino acids less than in S protein of SARS-CoV. This net charge shift to the positive side in $\mathrm{S}$ protein of SARS-CoV-2 was suggested to affect the capability for cell adhesion and help cross blood-brain barrier (BBB) (Bongrand 1998; Giulio Sancini et al. 2013). The viral entry into the host cell is mediated in two basic steps: 1). Viral spike spike. b The spike protein structure with furin cleave site and other components. RBD is as above. S1/S2 is the junction of the $\mathrm{S} 1$ and S2 domains. S2' contains a furin cleavage site with the unique amino acid sequence PRRA present in SARS-CoV-2. FP is the fusion peptide, HR1 and HR2 are heptad repeats, TM is a transmembrane domain. (a is created with BioRender.com)

attachment with the host receptor on the cell membrane and subsequent fusion of plasma membranes. This is facilitated by the ACE2 receptor and TMPRSS2 protease. 2). Fusion of the viral envelope membrane with host endosome/lysosome. This step is facilitated by cathepsin L, which can also compensate for cells that lack TMPRSS2 (Harrison et al. 2020).

\section{Vulnerability of the central nervous system for coronaviruses}

The coronaviruses primarily infect the epithelial cells in the respiratory tract. Some of these viruses like SARSCoV-1, MERS-CoV, and HCoV-OC43 elicited symptoms in the neuronal tissues (Bergmann et al. 2006; Iadecola et al. 2020; Huang et al. 2020a). This suggested that like other SARS viruses, SARS-CoV-2 may also infect the brain. SARS-CoV-2 trans-mucosally invaded from olfactory epithelium into the brain regions receiving olfactory projections (Meinhardt et al. 2021). The tissue expression pattern of coronavirus receptors (ACE2, BSG, NRP1) and proteases 
(TMPRSS2, TMPRSS11A, TMPRSS11B, Furin) in excitatory and inhibitory neurons, astrocytes, oligodendrocytes, microglia, and in cranial vascular endothelial cells is an indicator for SARS-CoV-2's vulnerability for these cells (Fig. 2) (Iadecola et al. 2020; Chen 2020).

The canonical receptor for $\beta$-coronavirus is ACE2. It binds with $\mathrm{S}$ protein of SARS-CoV but not of MERS-CoV. However, many non-canonical receptors can also help the entry of SARS-CoV-2 into the host cells. ACE2's primary function is to convert angiotensin II (Ang II) to Ang 1-7. It regulates cardiac function and many other functions in organs through the renin-angiotensin system. ACE2 also helps endocytosis of SARS-CoV-2 by binding to $S$ protein. ACE2 expression is higher in the small intestine, heart, testis, kidney, and thyroid and is medium in the lung, large intestine, bladder, liver, adrenal glands, and minimum in bone marrow, spleen, blood, and brain (Li et al. 2020a). ACE2 is shed in the bronchial space that can also bind the virus but it may not give access to the cells. The excitatory neurons have the highest ACE2 expression (Iadecola et al. 2020). It is also highly expressed in the central glial substance bordering the brain ventricles. This locus and through cerebrospinal fluid (CSF) route render a conduit for the CNS wide infection of SARS-CoV-2. The ACE2 is also expressed in the hippocampus. ACE2 receptor is not found in microglia, endothelial cells, and pericytes of the human brain and in a limited level expressed in the hippocampus. Therefore, SARS-CoV-2 infection in some of these cells or regions utilizes an alternate non-canonical receptor.

Neuropilin (NRP) is another non-conical receptor possibly utilized by the SARS-CoV-2. NRP has two family members, NRP1 and NRP2. It is a transmembrane receptor and binds to a substrate cleaved by furin. NRP1 is expressed in the olfactory epithelium and endothelial cells, respiratory epithelium, excitatory neurons. Its co-expression with ACE2 and TMPRSS2 in the olfactory epithelium and can markedly potentiate SARS-COV-2 entry in the CNS. Its extracellular b1/b2 domain binds with polybasic sequence PRRAR on the S protein of SARS-CoV-2. Another candidate is a basic immunoglobulin Basigin (BSG, CD147). It is a transmembrane glycoprotein expressed more in brain tissues than in the lung. Its expression in the embryonic kidney is very high. Dipeptidyl peptidase 4 (DPP4) is another non-canonical receptor, also known as CD26, implicated in COVID-19. It is an ectopeptidase. Conflicting data exist on its ability to bind the S protein of SARS-CoV-2 (Zhou et al.

SARS-CoV-2 infection effect

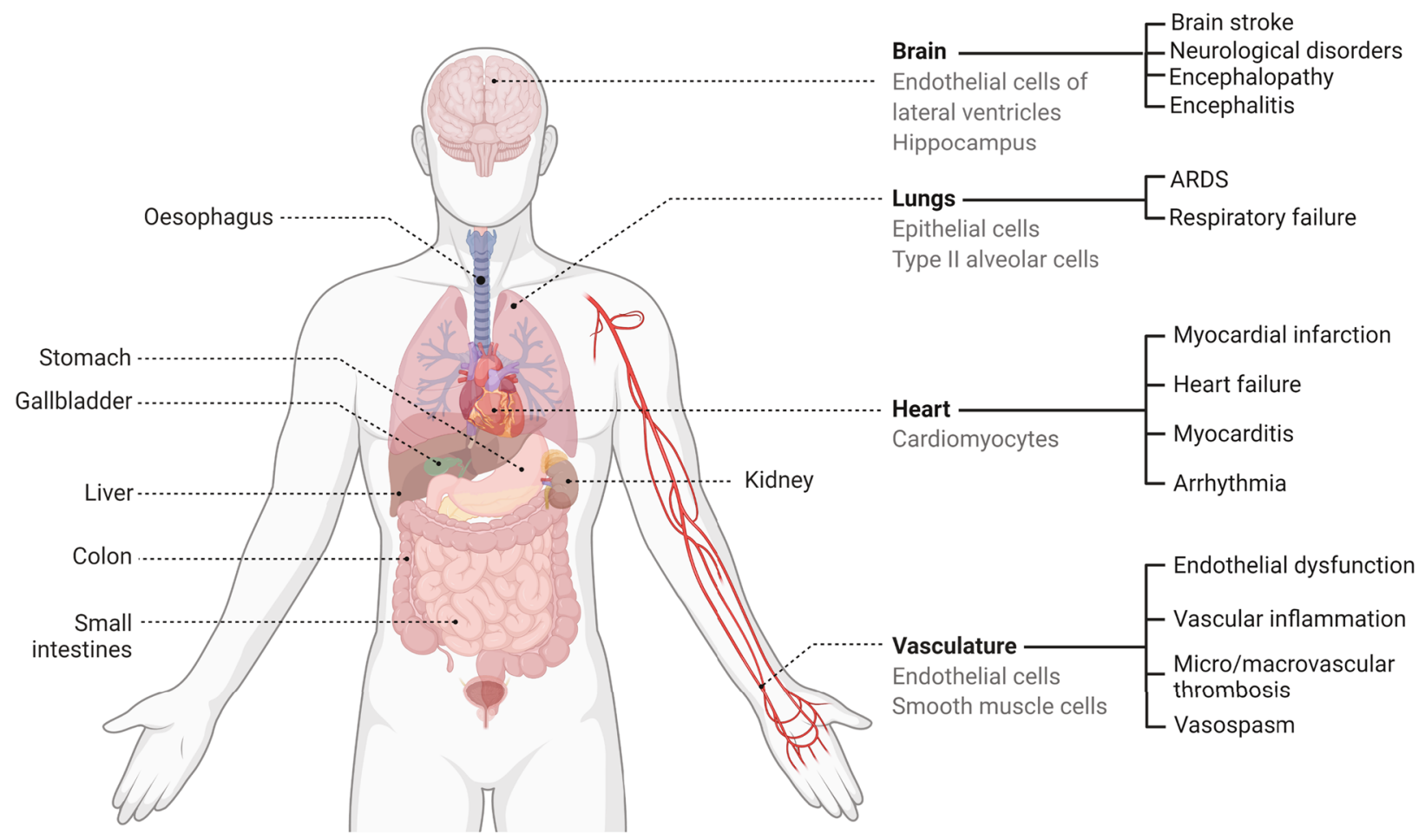

Fig. 2 The ACE2 receptor-expressing tissues and organs and effect of SARS-CoV-2 infection in these targets. The SARS-CoV-2 infection affects mainly the lung with ARDS and respiratory failure. The brain is also affected by stroke and encephalopathy. The encephalopathy may be due to encephalitis, which is not completely established. The vascular endothelial cells are damaged in COVID-19 patients and it could affect many tissues and organs. The detailed effects are listed in the figure. (The image is created with BioRender.com) 
2020a; Vankadari and Wilce 2020; Qi et al. 2020). Since both ACE2 and DPP4 have a similar expression pattern and bind to MERS-CoV, DPP4 may also bind to SARS-CoV-2. Alanyl aminopeptidase (ANPEP) is yet another potential receptor for SARS-CoV-2 because it helps viral entry of several coronaviruses including HCoV-229E into cells (Yeager et al. 1992). Similarly, glutamyl aminopeptidase (ENPEP) is also a potential receptor for SARS-CoV-2 because its expression pattern is very similar to the ACE2 but there is no definitive data available demonstrating its use for coronavirus entry (Qi et al. 2020). The angiotensin II receptor type 2 (AGTR2) is a G-protein coupled receptor (GPCR) and is highly expressed in the lung and binds to $\mathrm{S}$ protein with higher affinity than ACE2 (Cui et al. 2021).

In some areas of the brain BSG and NRP1 are more expressed than ACE2. The highest expression of BSG is in cranial endothelial cells and pericytes. NRP1 is highly expressed in excitatory neurons. Cranial endothelial cells express NRP1 compared to other brain areas. NRP1 is a molecule involved in axon guidance (Pang et al. 2014), angiogenesis, and vascular permeability (Plein et al. 2014). This indicates that SARS-CoV-2 has adapted a unique mode for viral entry by exploiting NRP1 (Moutal et al. 2021).

The TMPRSS11A and furin are more expressed in excitatory neurons than in inhibitory neurons albeit at low levels. SARS-CoV-2 shows a high propensity for excitatory neurons like in olfactory receptor neurons (ORN) and taste buds as evidenced by lack of smell and taste. Nevertheless, infection in inhibitory neurons of higher brain areas such as ventral tegmental area (VTA) and substantia nigra pars compacta $(\mathrm{SNpc})$ was reported. These areas are involved in cognition, working memory, and decision making. These structures also express ACE2 (Hernández et al. 2021). ACE2 is also expressed in the dorsal raphe nucleus (DRN) that expresses serotonin. DRN is involved in depression and anxiety, and it showed association with suicide cases in patients (Underwood et al. 1999). In summary, SARS-CoV-2 is prone to both excitatory and inhibitory neural circuits in the brain. This may be reflected in the serious psychological effects seen in patients with the advanced stages of COVID-19 (Helms et al. 2020a) because the psychological disorders arise out of imbalanced signals in the excitatory and inhibitory neural circuits.

SARS-CoV-2's capability to infect the nervous system using the ACE2 receptor was demonstrated (Song et al. 2021). However, SARS-CoV-2 has shown high susceptibility for dopaminergic neurons, which were derived from human pluripotent stem cells (that expressed low levels of ACE2) than cortical neurons and microglial cells (Yang et al. 2020). However, whether this was a primary infection in the neurons or a secondary invasion through other routes was not discerned. The viral infection in the brain and the development of encephalopathy or encephalitis warrants the presence of the virus in the brain or CSF. But corroborating substantial evidence was lacking in many cases of encephalopathy and encephalitis that could be directly implicated with SARS-CoV-2 infection (Bernard-Valnet et al. 2020; Ye et al. 2020). Nevertheless, a low level of viral RNA was detected in some post-mortem tissue of COVID-19 cases (Moriguchi et al. 2020; Solomon et al. 2020), though viral RNA was suspected of blood origin (Solomon et al. 2020). Therefore, implying that SARS-CoV-2's primary infection in the brain cause encephalopathy or encephalitis remains inconclusive.

The SARS-CoV-2's neuro-invasion primary evidence was obtained from human induced pluripotent stem cells (iPSC) derived neural progenitor cells (Song et al. 2021). The demonstration of SARS-CoV-2 replication in these cells strongly supported a neuro-invasion of the virus. This study also showed the presence of SARS-CoV-2 in the cortical regions of post-mortem brains of the COVID-19 patients with encephalopathy. However, an interferon (IFN) response was not detected, which was also true for organoidderived neural progenitor cells. Notably, the ACE2 receptor was utilized for infection in the organoids. The humanized mice infected via intracerebroventricular injections in the brain lost weight and died on the 6th day of post-infection. Remarkably, the human brain regions infected with SARSCoV-2 had no sign of lymphocyte or leukocyte infiltration. It was suggested that though the virus is neurotropic it did not trigger an immune response as seen with other neurotropic viruses such as Zika, rabies, herpes. The infected organoid-derived neurons created a local hypoxic environment. A similar condition arising in the brain might affect vasculature and lead to ischemic infarct that causes further infection of the tissue.

\section{The olfactory route for viral entry into the brain}

The olfactory route of viral entry was early on suspected in the COVID-19 outbreak because of the anosmia symptom noted in the patients (Politi et al. 2020). This possibility was further supported by the demonstration of the ACE2 receptor and TMPRSS2 in epithelial cells of the nasal mucosa. However, these molecules were not seen in the olfactory neurons (Meinhardt et al. 2021; Brann et al. 2020). Therefore, a direct entry of SARS-CoV-2 into the olfactory neurons is debatable. Nevertheless, the anosmia indicated a credible insult to the olfactory neurons during COVID-19. If olfactory neurons are infected, through the olfactory bulb the virus can reach the forebrain. The olfactory epithelium has $>50$ million neurons. These neurons have multiple cilia on each cell and each neuron expresses one type of olfactory receptor $(\mathrm{OR})$ in the cilium which is protruding from the nasal epithelium. The OR neuron axon projects to the olfactory glomerulus in the olfactory bulb. Here, ORs synapse 
with either mitral or tufted cells. Mitral cells project to five regions in the olfactory cortex: anterior olfactory nucleus, olfactory tubercle, piriform cortex, amygdala, entorhinal cortex. Tuft cells also project to the first two of the five regions. Among the olfactory cortical regions, except the first the rest of them are connected with the thalamus and frontal cortex. The thalamus sends projections to the orbitofrontal cortex, which along with the frontal cortex performs conscious discrimination of smell. In addition, the amygdala sends projections to the hypothalamus and the entorhinal cortex sends projections to the hippocampus (Masuo et al. 2021). Thus, the virus may even reach the cortex by transsynaptic traffic (Meinhardt et al. 2021), as reported for other viruses (Zingg et al. 2020).

\section{The systemic circulation and immune reaction for viral entry into the brain}

The alternate route for virus entry into the nervous system is through systemic blood circulation. Viruses in the bloodstream are known to cross the BBB (Bergmann et al. 2006). In some COVID-19 blood samples SARS-CoV-2 was detected (Zheng et al. 2020a). The virus requires molecular machineries of endocytosis and cellular trafficking to cross the BBB. The NRP1 and BSG receptors on cranial vasculature may facilitate the viral entry into the brain (CantutiCastelvetri et al. 2020). Another possibility is the disruption of the tissue integrity due to the inflammatory reaction elicited by the viral infection. The cytokines and interleukin-6 (IL-6), IL $\beta$, tumor necrosis factor (TNF), and IL-17 could damage the integrity of the BBB and the virus permeates into the brain (Erickson and Banks 2018). This effect is evident in the patients with comorbidities because an increased viral load in the brain was seen (Paniz-Mondolfi et al. 2020). For example, in Parkinson's disease, the SARS$\mathrm{CoV}-2$ virus was detected in the cerebral micro-vessels and neurons (Paniz-Mondolfi et al. 2020). This could be because the micro-vessels have been damaged. However, even with similar and other comorbidities, the virus was not always detected in certain brain samples. This could be because of technical limitations but otherwise it is quite enigmatic.

One more plausible way for SARS-CoV-2 to invade the brain is via hypothalamic median eminence because here the micro-vessels and tanycytes of median eminence express ACE2 and TMPRSS. These are used for docking and engulfing SARS-CoV-2 into neurons (Lazartigues et al. 2020). The virus can also invade immune cells and surreptitiously enter the brain. The monocytes, neutrophils, and T-cells can be the carrier for viruses and invade the brain tissue (Koyuncu et al. 2013). Coronavirus-like HCoV-229-E and HCoVOC43 could infect monocyte and macrophages. However, $\mathrm{HCoV}-\mathrm{OC} 43$ replication was restrained (Desforges et al. 2007). The coronavirus strains SK and SD isolated from the brain autopsy of multiple sclerosis patients were serologically related to the murine coronavirus A59 and $\mathrm{HCoV}$ OC43 (Gerdes et al. 1981). Likewise, SARS-CoV-2 may infect leukocytes and reach CNS by the 'Trojan horse' mechanism commonly deployed by many viruses (Huang et al. 2020a). However, the evidence for this route in COVID-19 is yet to be presented. Though the immune cells $\left(\mathrm{CD}^{+} 8^{+}\right)$in lymphoid organs (Zeqing Feng et al. 2020) and macrophages in broncho-alveoli carried SARS-CoV-2 components (Bost et al. 2020), it is unclear from where the viral particles were acquired by these cells (Merad and Martin 2020). The lack of immune cell infiltration in many autopsy samples suggested that lymphoid may not be primarily infected (Kantonen et al. 2020; Reichard et al. 2020). In summary, these findings indicate that SARS-CoV-2 can gain access into the nervous system via multiple routes though the exact trail of viral transmission into the CNS is unknown.

\section{Neurological effects in COVID-19 patients}

\section{Effects contributed by systemic factors}

\section{Maladaptive immune response}

The SARS-CoV-2 infection upregulates interferon-stimulated genes (ISGs), which activate IFNs. In severe cases of COVID-19, IFN $\alpha$ was high as in the case of SARS but IFN $\beta$ was not detected. Even the elevated IFN $\alpha$ and IFN $\lambda$ were subsided in mild and moderate cases of COVID-19. In severe cases, these again increased in the second week (Shen et al. 2020; Schultze and Aschenbrenner 2021). This pointed out the fact that SARS-CoV-2 could hijack the type 1 IFN response to evade the host's innate immunity. This is likely mediated by several mechanisms including, viral non-structural protein-16 (NSP16) mediated suppression of global mRNA splicing that could reduce viral RNA recognition (Banerjee et al. 2020). NSP1 can interfere with the 18S ribosomes to block the viral RNA translation. NSP8 and NSP9 block the protein trafficking to the plasma membrane. These multilevel actions leave the host unresponsive for initiating the anti-viral defense mechanism. In severe cases following the hyperactive phase of the immune response, the markers of immune suppression, like, kynurenines were elevated (Shen et al. 2020; Shu et al. 2020). In contrary, lipids including sphingolipids, glycerophospholipids, choline utilized in the immune response were reduced. While the complement system is activated by SARS-CoV-2 infections in COVID-19 patients as an early immune response, the platelet-derived chemokines are reduced. Collectively, in the severe cases of COVID-19, an early maladapted immune response was observed with elevated pro-inflammatory markers followed by suppression of the innate immune response. 


\section{Immunosuppression}

Generally, the viral entry in the cells triggers the type 1 IFN pathway. However, IFN $\beta$ is not expressed in the case of SARS-CoV-2 infection (Hadjadj et al. 2020). Normally, viral infection triggers the translocation of interferon regulator factor 3 (IRF3) - a transcription factor- into the nucleus and it drives IFN $\beta$ expression. This signalling appears to be hijacked by SARS-CoV-2. IFN is a positive feedforward signal for Signal Transducer and Activator of Transcription-3 (STAT3) pathway for anti-viral action in the cell. In inflammatory neurological diseases, such as encephalitis, higher levels of IL-6, IL-10, IL-12, chemokine ligand $C-X-C$ motif chemokine receptor $(\mathrm{CXC})$ motif ligand, CXCL8, and CXCL10 are detected in the CSF. On the other hand, in encephalopathy, IL-6, CXCL8, and active TGF $\beta 1$ were high in serum (Espindola et al. 2021).

During many viral infections, some viral particles are shed in the circulation. They act as the pathogen-associated molecular patterns (PAMPs). Often the infection can damage the tissues and releases a nuclear protein called high mobility group box 1 (HMGB1). It can work as the damageassociated molecular patterns (DAMPs). These molecules can gain entry into the brain if BBB integrity is compromised by cytokines, which is a possibility in COVID-19 infections. In the brain, these molecules trigger an innate immune response in pericytes, macrophages, and microglia through the Toll-Like Receptors (TLRs). TLRs via nuclear factor kappa-light-chain-enhancer of activated B cells (NF$\mathrm{kB})$ mediates the pro-inflammatory response by producing cytokines against the pathogens. An increased type 1 IFN response is seen in COVID-19 (Lee and Shin 2020). In severe cases, this immune reaction can indirectly damage brain cells and affect their functioning.

The lung is the first organ to be affected in COVID-19 because the virus predominantly affects the pneumocytes and proliferating epithelial cells (Carsana et al. 2020). The respiratory failure in COVID-19 leads to hypoxia (Kantonen et al. 2020). Alternatively, brainstem infection may affect the breathing center and respiration (Li et al. 2020b). This eventually damages the brain centers including the neocortex, hippocampus and cerebellum (Solomon et al. 2020). The induction of a cytokine storm is a major issue in COVID19 that eventually impacts other organ systems (Moore and June 2020; Fajgenbaum and June 2020; Qin et al. 2020). The cytokine storm may not spare the nervous system. The COVID-19 patients showed high titers of IL-2, IL-6, IL-8, IL-17, IL- $\beta$, TNF, granulocyte colony-stimulating factor (G-CSF), granulocyte-macrophage colony-stimulating (GM-CSF) factor, interferon gamma-induced protein 10 (IP10), monocyte chemoattractant protein-1 (MCP-1), and MCP $1 \alpha 2$. The IL- 6 and TNF levels are an indicator of the disease severity in COVID-19 (Diao et al. 2020). These molecules are released from the damaged cells against the virus. These signaling molecules form other molecular complexes with MGB1. Such molecular complexes could disrupt BBB and gain access to the brain (Chen et al. 2020b). This could elicit pathogen-associated and damage-associated responses in the brain via resident immune cells. This type of immune response uses TLR (Luan et al. 2016). The secreted S-protein could trigger TLR2, which in turn acted on NF-kB for activation of pro-inflammatory response in macrophage in SARS (Dosch et al. 2009). Similar humoral factors release affected brain function (Barakat et al. 2014). Though such immune responses were mounted immediately to battle SARS-CoV-2, it may lead to secondary damage in the brain and cognitive functions. However, substantiating data are required to verify this.

The hypothalamus-pituitary-adrenocortical (HPA) axis is highly sensitive to many humoral factors released in COVID-19 patients. The IL-6, IL- $1 \beta$, and TNF are major activators of the HPA axis (Dantzer 2018). This axis has a key role in regulating the proinflammatory response and suppression of the immune response by releasing the glucocorticoids (Smith et al. 1999). In the event of BBB damage in COVID-19 patients, the HPA axis could be activated, which may lead to neutrophilia (Cavalcante-Silva 2021) and lymphopenia (Huang and Pranata 2020).

Hyper coagulation was frequently reported in COVID-19 patients (Helms et al. 2020b; Perico et al. 2021). They had increased fibrinogen and D-dimers and prolonged clotting time. It might have been facilitated by the pro-coagulant IL-6. Intravascular thrombosis could also affect the brain (Bolaji et al. 2020). In COVID-19, multi-organ failure was often reported (Lopes-Pacheco 2021), which could indirectly affect brain function. For example, the patients with cardiac dysfunction developed a higher rate of thromboembolic events and ischemic strokes $(\sim 1.6 \%)$ that affected brain function in some COVID-19 patients (Inciardi et al. 2020; Merkler et al. 2019).

\section{Encephalitis and encephalopathy}

Among the hospitalized COVID-19 patients $30 \%$ had neurological abnormalities (Helms et al. 2020c). This is increased to $45 \%$ in patients with severe respiratory distress and $85 \%$ in patients with acute respiratory distress syndrome (ARDS). Mild neurological symptoms like headache, nausea, dizziness, and malaise, loss of smell and taste were seen in a majority of the patients. In the serious cases of COVID-19 patients, the neurological symptoms were severe, indicative of encephalopathy and encephalitis. In encephalopathy mental confusion, disorientation, agitation, and sleeplessness have been seen in $<5 \%$ of the COVID-19 patients (Mao et al. 2020). However, it was commonly reported in patients with ARDS (Helms et al. 2020c). Encephalitis is inflammation 
of the brain tissue following infection. It is unclear whether encephalitis leads to encephalopathy in the COVID-19 patients. In many cases of encephalopathy classic symptoms of encephalitis like fever, seizure, mental status perturbed, white blood cells (WBC) in CSF were noted along with the brain damage as visible on magnetic resonance imaging (MRI) (Venkatesan et al. 2013). However, in very rare cases CSF had the presence of virus (Huang et al. 2020b; Moriguchi et al. 2020). In one case, perivascular lymphocyte infiltrates and neuronal damage was seen in encephalitis but the virus could not be detected (Efe et al. 2020). These COVID-19 patients developed critical conditions due to hypoxia, hypotension, renal failure, heavy sedative administration, extended immobility, and isolation. These were also predisposing factors to develop encephalopathy (Cummings et al. 2020; Maas 2020). The lack of evidence for SARS-CoV-2 in the brain and very few encephalitis cases in patients of COVID-19 suggests that encephalopathy may be due to secondary effects of viral infection.

\section{Stroke and brain tissue damage}

The occurrence of stroke in COVID-19 patients was 1 to $3 \%$ in hospitalized cases and 6\% in critical cases (Merkler et al. 2019). Most often the stroke was due to the lodging of a blood clot in the brain vessels that caused the embolic stroke. The incidence of embolic stroke was higher in the older population with vascular comorbidities. The brain stroke may not be caused by a viral infection but by a secondary cause. Acute cardiac injury in COVID-19 patients could cause cardiac failure and brain damage. Thus, brain stroke may also have a primary cause originating in damaged vascular tissue.

The incidence of thrombotic events was higher in COVID-19 patients than in patients with other respiratory viral diseases (Bilaloglu et al. 2020). The overall incidence of venous thrombotic events was $8 \%$ in COVID-19 patients and it rose to $69 \%$ in the case of critically ill patients (Moores et al. 2020). Another study reported that 20-55\% of the hospitalized COVID-19 patients had coagulopathy with doubled D-dimer values and 1-3 s prolonged prothrombin time (Lee et al. 2020). Another study reported a three to four-fold increase in D-dimer in COVID-19 patients with a poor outcome (Rostami and Mansouritorghabeh 2020) A higher level of D-dimer is a sign of hyper coagulopathy and it can cause an increased rate of cerebrovascular events and stroke in COVID-19 patients. However, more definitive evidence, including determination of the molecular mechanisms, is required to unequivocally establish the link between hyper coagulopathy and COVID-19. Thrombosis was high in lung (16.5\%) and brain (14.8\%) of COVID-19 patients (Suh et al. 2021; Hoelscher 2020). Intriguingly, increased prothrombin time and D-dimer (fibrin degradation product) were associated with higher mortality in COVID19 patients (Wang et al. 2020b).

Stroke was mainly reported in older COVID-19 patients (Oxley 2020; Klein 2020; Lodigiani et al. 2020). Patients with a median age of 66 years had $7.7 \%$ thrombotic events and half of them developed thrombotic events within $24 \mathrm{~h}$ of hospital admission (Lodigiani et al. 2020). The strokerelated mortality was $67 \%$ higher in COVID-19 patients with age 57-70 years compared to $>70$ years age (Fridman et al. 2020). The large vessel occlusion incidences doubled (46.9\%) in COVID-19 patients. Regardless of the age and comorbidities, large vessel occlusion incidences were higher in COVID-19 patients. This indicates that COVID19 patients have increased hypercoagulability even without comorbidities. However, the risk of mortality increased with the coincidence of old age, cardiovascular comorbidity, and severe COVID-19.

It is notable that in COVID-19 patients $22 \%$ had a cardioembolic stroke and a large majority $(\sim 65.6 \%)$ had a cryptogenic stroke (Yaghi et al. 2020). In this context, it should be noted that in the initial days of COVID-19 a thorough investigation on the cause of the stroke was unable to be determined. It was suggested that the cryptogenic stroke might be due to hypercoagulability. In COVID-19 patients, the reduced respiratory function results in hypoxia, a trigger for sympathetic nervous system stimulation of the heart. This can result in cardiac arrhythmia and cardiomyopathy. Under such conditions, increased hyper coagulopathy may be a compounding risk factor for cardioembolic stroke. The cardioemboli might be suspected in stroke if infarction is detected in multiple cerebral vascular vessels or with systemic and cerebral emboli, ventricular dyskinesia, atrial fibrillation and right to left cardiac shunt. Cardioembolic events associated with COVID-19 might be a cause for stroke. The intravenous immunoglobulins used for COVID19 treatment may also cause a stroke (Venketasubramanian and Hennerici 2020).

In COVID-19 patients, haemorrhagic stroke was lower compared to ischemic stroke (Spence et al. 2020). The ischemic stroke in COVID-19 patients may be due to hypercoagulability, vasculitis, and cardiomyopathy. The haemorrhagic stroke may be due to the rupture of the endothelial cells of the cranial vessel that express the ACE2 receptor. The cytokine storm also can damage vessels and cause haemorrhagic stroke.

COVID-19 patients often required hospitalization and invasive mechanical ventilation. In a clinical setting, $11.9 \%$ of such cases had bacteraemia, which was lower $(1.8 \%)$ in the case of non-invasive ventilation (Goyal et al. 2020). In another setting, $7.1 \%$ of COVID-19 patients' blood cultures detected bacteria (Hughes et al. 2020). Thus, in addition to the primary viral infection in COVID-19 patients, secondary bacteraemia frequently develops, which can lead to 
18-fold increase in myocardial infarction and stroke (Dalager-Pedersen et al. 2014). Septic emboli were detected in $10 \%$ of COVID-19 cases with brain haemorrhage (Coolen et al. 2020).

Patients who required ICU had a $31 \%$ frequency of thrombotic events (Klok et al. 2020). Venous thrombo emboli (VTE) were frequent in COVID-19 and reported in $1 / 3(\sim 30 \%$ only) of the patients. It was associated with increased severity of the disease and mortality. The majority had pulmonary emboli. The VTE was high (16 to 42\%) depending on the day post-infection, and in ICU patients it was further aggravated to 26-59\% (Middeldorp et al. 2020).

The coagulopathy most often started in the lungs in cases of severe COVID-19. However, it can also be due to pro-coagulant action of IL-6, endothelial damage (Goshua et al. 2020), complement activation (Ramlall et al. 2020), and neutrophil recruitment. The neutrophils from neutrophil extracellular traps (NETs) with decondensed chromatin with histones are released into the circulation (Middleton et al. 2020). The NETs produced by programmed cell death are called NETosis. The NETs trap and kill pathogens. The NETosis induced by platelets can be dysregulated and result in tissue damage, hypercoagulability, and thrombosis. In COVID-19 plasma contained platelet factor 4 (PF4) and Regulated upon Activation, Normal T-Cell Expressed and Presumably Secreted (RANTES) (Middleton et al. 2020). PF4 and RANTES are inducers for NETosis (Rossaint et al. 2014; Hundelshausen et al. 2005).

\section{Multi-organ failure and its impacts on COVID-19 outcomes}

In COVID-19 the major organs affected are the lung, brain, heart, liver and kidneys (Mokhtari et al. 2020). Lung inflammation causes ARDS and leads to hypoxia, which can affect brain centres for chemoreception, heartbeat, and breathing. These responses are necessary to cope with the low percent saturation of oxygen in the blood $\left(\mathrm{SpO}_{2}\right)$ during ARDS. ACE2 is highly expressed in type II alveolar cells of the lung permitting SARS-CoV-2 infection and leading to lung inflammation and injury. Pneumonitis developed is of a pauci-inflammatory type with a septal capillary mural, luminal fibrin deposition, capillary injury, and neutrophils infiltration in the inter-alveolar septa (Magro et al. 2020). Along with this condition, C5b-9 (membrane attack complex), C4d, and mannose-binding lectin (MBL)-associated serine protease (MASP) 2 were upregulated in the microvasculature, a sign of systemic activation of the complement pathways (Magro et al. 2020).

ACE2 receptors are also present in the heart (Zou et al. 2020), allowing viral infection, which can cause myocarditis and lead to heart failure and sudden death. Therefore, viremia is possible in the heart. Among COVID-19 patients,
$12 \%$ without previous history of cardiovascular disease (CVD) had increased troponin I (a marker for myocardial ischemia or necrosis) or cardiac arrest in the hospitalized state (Zheng et al. 2020b). Cardiac conditions in COVID19 were associated with elevated C-reactive protein (CRP) levels, pneumonia, old age, and other underlying comorbidities (Xu 2020). Cytokine storm can also cause cardiac damage by infiltration of inflammatory cells (Zhang et al. 2016). Fibrosis and cardiomyocyte hypertrophy were very frequently seen in COVID-19 and it may be due to the side effects of the cytokine storm, endothelial dysfunction, leukocyte infiltration, and microvascular thrombosis (Bradley et al. 2020).

Liver biochemistry can become irregular in COVID-19 patients. The level of liver function enzymes such as lactate dehydrogenase (LDH), aspartate transaminase (AST), alanine transaminase (ALT), total bilirubin (TB), alkaline phosphatase (ALP) was significantly higher in COVID-19 patients (Fan et al. 2020). A high AST was strongly correlated with higher mortality in COVID-19 patients (Lei et al. 2020). CRP and serum ferritin were increased in COVID19 patients (Liu et al. 2020). Bile duct cells have a higher expression of ACE2 than other liver cells (Banales et al. 2019). In the case of SARS, SARS-CoV titre was low in the liver (Chau et al. 2004) but SARS-CoV-2 was not detected in a liver sample of a COVID-19 post-mortem case (Xu et al. 2020), and evidence for liver infection was not available (Marjot et al. 2021). The main finding in COVID-19 patients was hepatic steatosis; a sign of sepsis (Medeiros et al. 2020). Hepatic injury due to smooth muscle fragmentation in portal vein was reported in COVID-19 patients (Wang et al. 2020c). Liver injury decreased the platelet count and activated fibrinolytic and coagulative pathways (Wang et al. 2020c). The drugs used during COVID-19 may also induce liver damage independent of infection-induced damages. This could come from steroidal, non-steroidal, and herbal remedies ( $\mathrm{Li}$ and Xiao 2020). The acute kidney infection was $29 \%$ in severe cases and $69.57 \%$ in older age patients (Diao et al. 2021) and it increased mortality 5.3 times compared to 1.5 times for chronic diseases (Zhen Li et al. 2020; Su et al. 2020). The mortality was associated with increased proteinuria, higher creatinine levels, blood urea nitrogen, and haematuria (Cheng et al. 2020).

Neurological diseases were highly reported in COVID-19 patients admitted to ICU. The severe patients had indications of CNS infection/damage and in mild to moderate cases had indications of peripheral nervous system (PNS) (Mao et al. 2020). The CNS-related symptoms were headache, dizziness, ataxia, acute cerebrovascular disease, epilepsy, and altered consciousness. The symptoms like hypoplasia, neuralgia, fatigue, myalgia, anosmia (or hyposmia), and hypogeusia were associated with the PNS origin (Mao et al. 2020). A case of acute necrotizing encephalopathy 
had been reported in a COVID-19 positive patient, and the virus was detected in CSF after 19 days post symptoms and the patient tested was negative for SARS-CoV-2 (Virhammar et al. 2020). It may be the degraded fragments of viral RNA. One COVID-19 patient presented with seizures and further investigation detected demyelination in periventricular white matter and the bulbo-medullary junction of the brain. However, SARS-CoV-2 RNA was not traceable in the CSF (Zanin et al. 2020). A paediatric case of encephalitis was reported with COVID-19 (McAbee et al. 2020).

The neurological pathogenesis of COVID-19 has two origins: (1). the infection of nerve endings like in olfactory sensory neurons and the retrograde trafficking of the virus into the brain. (2). cytokine storm and viral sepsis elicited neural damages (Meinhardt et al. 2021). The virus transmission from the olfactory route to the medulla oblongata in the brain stem can affect the cardio-respiratory regulatory centre. Axonal injuries, microscopic brain necrosis, and infarcts were seen in COVID-19 patients (Reichard et al. 2020). As mentioned early, cytokine storm can indirectly disrupt the BBB (Mehta et al. 2020), and cause coagulopathies leading to brain dysfunction and stroke ( $\mathrm{Li}$ et al. 2020c). All these neural damages aggravate the disease outcome in COVID19 patients.

\section{Chemo sensory loss}

The chemosensory loss was $~ 30-80 \%$ in COVID-19 patients (Reiter 2020). Many patients early on in the pandemic reported the loss of smell and taste but it was recovered in $75 \%$ of the patients. The fast recovery might be due to the ability of these neurons to regenerate, especially the taste receptors, which can regenerate within 2 weeks (Feng et al. 2014). While the olfactory neurons may survive 60 days or more (Sultan-Styne et al. 2009). Hence, if the infection in chemo-sensory neurons did not spread to the brain, it will not have any long-term effect on the CNS.

\section{The immune response elicited neuronal damage}

The unprecedented level of immune response after SARSCoV-2 infection can cause many secondary responses that can affect neural tissue function. This could necrotize neurons or hemorrhagic damages to the neurons. Such a possibility was indicated by the manifestation of Guillain-Barre Syndrome in patients of COVID-19 (Toscano et al. 2020). They have demyelination and weakness. In some patients, the demyelination was in a cranial nerve that was Miller Fisher variation of Guillain-Barre Syndrome. The direct involvement of SARS-CoV-2 was not demonstrated in these cases, again indicating a secondary effect of an immune response. This is supported by the presence of anti-ganglioside antibody (anti-GD1b) in the affected cranial nerve (Gutierrez-Ortiz et al. 2020).

\section{Intensive care related}

A large number of patients were admitted to intensive care and most often required ventilator support (Helms et al. 2020a; Cummings et al. 2020), with those requiring ventilators often developing delirium (Ely et al. 2001). In COVID19 patients who developed ARDS invariably required ventilators, were also susceptible to delirium. It might be due to low blood oxygen triggered hypoxia and/or sedation side effects affecting the brain. While those older patients with delirium had high mortality rates hence those with delirium required special care (Kennedy 2020).

\section{Psychiatric disturbances}

The patients infected with the SARS-CoV-1 developed psychiatric disturbances like depression, anxiety, insomnia. In a meta-analysis of the COVID-19 patients 34\% had insomnia, $45 \%$ had depression, and $47 \%$ had anxiety (Deng et al. 2021). The neurological symptoms in COVID-19 were in common with other respiratory influenza viral infections. The available data indicated that SARS-CoV-2 has no special propensity for infecting neural tissues like the neurotropic herpes simplex virus, which infects the brain. However, the furin cleavage sequence in SARS-CoV-2 is intriguing and it may determine the viral neurotropism (Cheng 2021). The first COVID-19 patient with a psychiatric disorder had maniclike symptoms, which developed even after vital signs were normal (Lu et al. 2020). In a suspected COVID-19 patient had a psychiatric disorder but without serious respiratory distress, it may render the virus neurotropic (Mawhinney et al. 2020). The neurotropism-driven neuropathy of SARS$\mathrm{CoV}-2$ is yet to be verified by more research. Given the enormity of cases in the COVID-19 pandemic, it can be easily investigated.

\section{The genetic susceptibility loci for adverse outcomes in COVID-19}

The GWAS analysis on the largest ever COVID-19 positive samples revealed the genetic architecture of COVID-19 patients (Initiative 2021). It has revealed 13 loci genomewide with a significant association for hospitalization and ICU cases. These loci have genes involved in lung function and type 1 inflammatory response. Among the genes, the top hit was TYK2 (tyrosine kinase 2), which is involved in the primary immune response of interferon- $\alpha$ (IFN- $\alpha$ ), interleukin-6 (IL-6), IL-10, and IL-12. TYK2 loss of function mutation resulted in primary immunodeficiency (Dendrou 
et al. 2016). The others were lung-specific variants of forkhead box P4 (FOXP4), interferon-alpha, and beta receptor subunit 2 (IFNAR2), 2'-5'-oligoadenylate synthetase 1 (OAS1) OAS-2, OAS-3, dipeptidyl peptidase9 (DPP9). FOXP4 is associated with lung adenocarcinoma (Dai et al. 2019). IFNAR2 is part of the innate immune response (Lutfalla et al. 1995). The OAS1, OAS2, and OAS3 are induced by IFNs and catalyse the synthesis of 2-prime,5-prime oligomers of adenosine (2-5As). 2-5As along with RNase L degrades the viral RNAs (Hovnanian et al. 1998). OAS1 was associated with infantile pulmonary alveolar proteinosis that resulted in dyspnoea (Cho et al. 2018). DPP9 is a serine protease with a role in cell adhesion and is a suggested candidate for pulmonary fibrosis (Initiative 2021; Zhou and Wang 2016; Fingerlin et al. 2013; Wang et al. 2021). The $A B O$ blood group locus has also shown a significant association with COVID-19 disease/severity (Wang et al. 2021; Initiative 2021). An intronic variant of lysine acetyltransferase 8 (KAT8) regulatory NSL (non-specific lethal) complex subunit 1 (KANSL1) was protective in COVID-19 cases because it reduced the requirement for hospitalization (Initiative 2021). KANSL1 functions on the histone for regulating gene expression and it has an association with reduced lung function (Smith et al. 2005).

A variant of CXCR6 showed a significant association with COVID-19 disease/severity. It is involved in chemokine signalling and is a co-receptor for some retroviruses (Matloubian et al. 2000). Leucine zipper transcription factor-like 1 (LZTFL1) is another gene in the same locus and its product is transiently associated with Bardet-Biedl syndrome (BBS) complex, which is a critical component required in cilia function (Seo 2011). Lung epithelium has numerous cilia per cell. Cilia dysfunction compromises lung function (Tilley et al. 2015). In olfactory neurons the receptors are hosted in cilia, in brain ventricles CSF is propelled by cilia (Fliegauf et al. 2007). Hence dysfunction of cilia may affect the lung and neural systems. A variant of the solute carrier family 6 member 20 (SLC6A20) increased adverse clinical presentation in COVID-19. SLC6A20 is a putative glycine transporter and has a function in the kidney (Broer et al. 2008). These findings suggested that the severity of the clinical outcome in COVID-19 patients may be determined by polygenic traits. Higher body mass index (BMI) increased the severity of the outcome but Type 2 diabetes was not a factor implicated in COVID-19 (Initiative 2021). The signalling pathways involved by these genetic loci could be targeted for therapeutic intervention in COVID-19 patients.

\section{Therapeutic considerations for COVID-19}

SARS-CoV-2 has no definitive drug to stop viral replication. Hence the strategic goal is to avoid getting infected, which is partially achieved by vaccines. The researched across the world developed various types of vaccines. Now $\sim 22$ vaccines are approved under emergency use approval (EUA) at least by one country (https://covid19.trackvaccines.org/). ZyCoV-D is a DNA vaccine in $\mathrm{Ph} 3$ trial in India. Moderna's mRNA-1273 is the first RNA vaccine developed and tested under EUA against for the SARS-CoV-2. Japan approved it under the name TAK-919. BNT162b2 is another RNA vaccine developed by Pfizer/BioNTech. ZF2001, CIGB66, MVC-COV1901 are a few of the protein subunit-based vaccines approved under EUA. Sputnik V, Ad26.COV2.S, AZD1222, Covishield, etc. are non-replicating viral vectorbased vaccines tested under EUA. Covaxin (India) and KoviVac (Russia), BBIBP-CorV (China) are the inactivated virus-based vaccines used under EUA.

Once infected the main focus of treatment is to reduce the viral load by generic antiviral drugs. The main anti-viral drug repurposed for treating the COVID-19 is remdesivir. It is ATP analog and interferes with RNA-dependent RNA polymerase and evades the viral exonuclease and is a broadspectrum anti-viral drug approved under EUA. However, WHO Solidarity Trial Consortium found little or no effect of remdesivir and anti-viral drugs for hospitalized COVDI19 patients (Consortium W.H.O.S.T 2021). The anti-viral repurposing for COVID-19 must be done with caution to avoid drugs that develop phospholipidosis, a toxicity confound, instead of truly targeting the virus (Tummino et al. 2021).

The next focus is to reduce respiratory insufficiency. The silent hypoxia may be the cause of multi-organ failure in COVID-19 patients (Wilkerson et al. 2020). The neurological outcomes in COVID-19 may also be due to hypoxic insult. The neurological manifestations in COVID-19 have no special treatment regimen other than adopting the standard procedures to manage the condition. Initially, hypoxia may not present any underlying clinical conditions but constant monitoring of $\mathrm{SpO}_{2}$ and maintaining $\mathrm{SpO}_{2}>95 \%$ is required to avoid emergency (Wilkerson et al. 2020).

The immune response must be checked before an overwhelming inflammatory response is elicited. As noted earlier, in certain cases of COVID-19 immune system may become hyperactive. To reduce its deleterious effect on the body, immune suppression is desired and the immunomodulation therapies achieve it to certain extent. Baricitinib (Olumiant) is an immunomodulator for COVID-19 and is given in combination with remdesivir. It is a Janus kinase (JAK) inhibitor. JAKs are intracellular enzymes which transmit signals arising from cytokine or growth factor-receptor 
interactions on the cellular membrane to influence cellular processes of hematopoiesis and immune cell function.

Actemra (Tocilizumab), a monoclonal antibody $(\mathrm{mAb})$ that reduces inflammation by blocking the interleukin-6 receptor and it does not target SARS-CoV-2. Approved for patients above 2 years of age. It is a recombinant humanized $\mathrm{mAb}$ that selectively targets soluble and membranebound human IL-6 receptors (sIL-6R and mIL-6R) and inhibits IL- 6-mediated signalling. It is administered for patients receiving systemic corticosteroids and require supplemental oxygen, non-invasive or invasive mechanical ventilation, or extracorporeal membrane oxygenation (ECMO).

The SARS-COV-2 can be neutralized by antibodies against its specific proteins. It can block viral entry into human cells. Therefore, utilization of mAbs generated against SARS-COV-2 has been explored for treatment. Four mAbs are approved under EUA for treating mild to moderate cases of COVID-19 in adults and paediatric patients above 12 years and with a minimum $40 \mathrm{~kg}$. REGEN-COV is a combination of casirivimab and imdevimab recombinant neutralizing human IgG1 monoclonal antibodies. They target the receptor binding domain (RBD) of the spike protein of SARS-CoV-2. Sotrovimab is also recombinant human IgG1 $\kappa$ mAbs. It targets a conserved epitope on RBD of SARS-CoV-2. The drug is not a competitor for human ACE2 receptor binding. Hence it will not block the endogenous ACE2 receptor. Bamlanivimab and etesevimab are used in combination and are IgG1 mAbs that target non-overlapping epitopes within the RBD of SARS-CoV-2.

The consequence of immune reaction is often leading to hyper coagulation, which also needs special attention to avoid thrombus and stroke. Thus, a multi-level symptomatic treatment initiated early in the disease may stop tissue damage and the rapid deterioration of the infected organs including the brain areas. The delay to check it may lead to multi-organ failure and fatality. The cytokine storm, DAMS, and PAMS reactions influence blood circulation including cranial circulation. The thromboembolic reactions further confound cranial blood circulation and cause the onset of stroke. Cytokine storm in serious cases of COVID-19 can be suppressed by a short course of corticosteroids for saving life (Kolilekas et al. 2020). However, it is not advised for mild cases of COVID-19 because early use of it can adversely affect the outcome (Group R.C et al. 2021). The short-term effect of symptomatic treatment is not much understood.

Patients $>70$ years were more prone to develop brain strokes (Fridman et al. 2020). Surprisingly, a cohort of patients did not show any sign of brain damage during the COVID-19 but later on developed brain damage (Kantonen et al. 2020). The major long-term impact of COVID-19 associated hypoxia or silent stroke will be on cognitive functions. The hypoxic shock either due to low $\mathrm{SpO}_{2}$ or disruption in local blood supply can damage neural function. Ischemic brain damage is a known cause of cognitive impairment (Pendlebury et al. 2019), and COVID-19 recovered patients showed cognitive deficits (Jaywant 2021). Among long-term effects, the COVID-19 patients are at higher risk to develop Alzheimer's disease (Hampshire et al. 2021; Heneka et al. 2020). In COVID-19 recovered patients a change in neural functions that was visible on electroencephalogram (EEG) (Kopanska et al. 2021). The CNS neurons, if damaged due to prolonged hypoxia, may be difficult to repair (Holmes 2017). So the long-term effects of COVID-19 due to neural damage and hypoxic insult in the CNS are unclear now. Follow-up of patients recovered from COVID-19 with neurobehavioral observations may shed light on any possible long-term effects of the CNS.

\section{Conclusions}

The novel $\beta$-coronavirus SARS-CoV-2 causing COVID-19 has emerged as the most devastating pandemic in a century. SARS-CoV-2 has a unique furin cleavage site in the spike protein that might result in the enhanced infectivity and neurotropism. Virus transmissibility was reduced by rapid vaccination and protected patients from developing severe clinical symptoms and an adverse outcome. However, the rapidly evolving viral mutations may evade antibodies developed by vaccination and result in breakthrough infections. Therefore, until an effective drug is available to inhibit SARS-CoV-2 infection, the possibility of seasonal re-emergence of COVID-19 cannot be eliminated. A large number of COVID-19 patients were hospitalized and required intensive care with ventilator support to combat the acute respiratory distress syndrome. The respiratory distress and hypoxia were the primary concern in COVID-19 patients. But in many patients the secondary health complications including neurological disorders like encephalitis, encephalopathy and subsequent multi-organ failure are serious challenges. The neurological symptoms have similarities to encephalitis but substantial evidence for primary virus invasion into the brain is lacking. The respiratory distress and hypoxia and/or the cerebrovascular failure elicited by the humoral immune factors released in the systemic circulation may be the alternative cause for the encephalopathy. The coagulopathy and thrombosis leading to embolic stroke is a major cause of brain damage leading to secondary health complications of neurological effects including delirium and manic psychiatric behaviors. Though the neurological damage is secondary in COVID-19 patients, the widespread incidence of the virus in society eventually will leave a large number of patients affected with encephalopathy and stroke. Given the knowledge gap in the mode of SARS-CoV-2 transmissibility into neurons and how it affects the brain more interdisciplinary basic research is warranted. A large number of brain 
samples available during the pandemic may be an invaluable resource for resolving this unmet challenge. The animal models of SARS-CoV-2 can be utilized to mimic human brain damage in COVID-19 patients, though it requires a humanized version of the ACE2 receptor. Such experiments pose a caveat and need a high level of biosafety measures. Besides COVID-19 patients, the post-pandemic subjects will also need long-term health observations, care assistance and attention. The new health care policies will have to factor in such long-term community goals for proper management of the COVID-19 pandemic outcome in the long run.

Acknowledgements The author acknowledges the grant support from ICMR-NIN [ST/1/Intra-Proj-Allot(1)/2020-21 dated 05, January, 2021; ST/1/Intra-Proj-Allot(3)/2020-21 dated 12, February, 2021]. The author also acknowledges Dr. Suneel Kateriya, SBT, JNU, New Delhi for help to create images. The author acknowledges the helpful suggestions given by the anonymous reviewers to improve the manuscript and Dr. Stephen Rawlinson, Monash University, Australia, for reading the manuscript and giving valuable suggestions.

Author contributions The review is conceived and written by SV.

Availability of data and material Not applicable.

Code availability Not applicable.

\section{Declarations}

Conflict of interest The author declares no conflict of interest/competing interests.

\section{References}

Andersen KG et al (2020) The proximal origin of SARS-CoV-2. Nat Med 26(4):450-452

Banales JM et al (2019) Cholangiocyte pathobiology. Nat Rev Gastroenterol Hepatol 16(5):269-281

Banerjee AK et al (2020) SARS-CoV-2 disrupts splicing, translation, and protein trafficking to suppress host defenses. Cell 183(5):1325-1339e 21

Barakat W et al (2014) Candesartan and glycyrrhizin ameliorate ischemic brain damage through downregulation of the TLR signaling cascade. Eur J Pharmacol 724:43-50

Bergmann CC, Lane TE, Stohlman SA (2006) Coronavirus infection of the central nervous system: host-virus stand-off. Nat Rev Microbiol 4(2): 121-132

Bernard-Valnet R et al (2020) Two patients with acute meningoencephalitis concomitant with SARS-CoV-2 infection. Eur J Neurol 27(9):e43-e44

Bilaloglu S et al (2020) Thrombosis in hospitalized patients with COVID-19 in a New York City Health System. JAMA 324(8):799-801

Bolaji P et al (2020) Extensive cerebral venous sinus thrombosis: a potential complication in a patient with COVID-19 disease. BMJ Case Rep 13(8):e236820

Bongrand P (1998) Specific and nonspecific interactions in cell biology. J Dispers Sci Technol 19(6-7):963
Bosch BJ et al (2003) The coronavirus spike protein is a class I virus fusion protein: structural and functional characterization of the fusion core complex. J Virol 77(16):8801-8811

Bost P et al (2020) Host-viral infection maps reveal signatures of severe COVID-19 patients. Cell 181(7):1475-1488e12

Bradley BT et al (2020) Histopathology and ultrastructural findings of fatal COVID-19 infections in Washington State: a case series. Lancet 396(10247):320-332

Brann DH et al (2020) Non-neuronal expression of SARS-CoV-2 entry genes in the olfactory system suggests mechanisms underlying COVID-19-associated anosmia. Sci Adv 6(31):eabc5801

Broer $\mathrm{S}$ et al (2008) Iminoglycinuria and hyperglycinuria are discrete human phenotypes resulting from complex mutations in proline and glycine transporters. J Clin Invest 118(12):3881-3892

Cantuti-Castelvetri L et al (2020) Neuropilin-1 facilitates SARS-CoV-2 cell entry and infectivity. Science 370(6518):856-860

Carsana L et al (2020) Pulmonary post-mortem findings in a series of COVID-19 cases from northern Italy: a two-centre descriptive study. Lancet Infect Dis 20(10):1135-1140

Cavalcante-Silva LHA et al (2021) Neutrophils and COVID-19: the road so far. Int Immunopharmacol 90:107233

Chau TN et al (2004) SARS-associated viral hepatitis caused by a novel coronavirus: report of three cases. Hepatology 39(2):302-310

Chen R et al (2020) The spatial and cell-type distribution of SARS$\mathrm{CoV}-2$ receptor ACE2 in the human and mouse brains. Front Neurol 11:573095

Chen L et al (2020a) Convalescent plasma as a potential therapy for COVID-19. Lancet Infect Dis 20(4):398-400

Chen L et al (2020b) Elevated serum levels of S100A8/A9 and HMGB1 at hospital admission are correlated with inferior clinical outcomes in COVID-19 patients. Cell Mol Immunol 17(9):992-994

Cheng J et al (2019) The S2 subunit of QX-type infectious bronchitis coronavirus spike protein is an essential determinant of neurotropism. Viruses 11(10):972

Cheng Y et al (2020) Kidney disease is associated with in-hospital death of patients with COVID-19. Kidney Int 97(5):829-838

Cheng J et al (2021) The furin-S2' site in avian coronavirus plays a key role in central nervous system damage progression. J Virol 95:e 02447

Cho K et al (2018) Heterozygous mutations in OAS1 cause infantileonset pulmonary alveolar proteinosis with hypogammaglobulinemia. Am J Hum Genet 102(3):480-486

Conde Cardona G et al (2020) Neurotropism of SARS-CoV 2: Mechanisms and manifestations. J Neurol Sci 412:116824

Consortium WHOST (2021) Repurposed antiviral drugs for Covid19 - interim WHO solidarity trial results. N Engl J Med 384(6):497-511

Coolen $\mathrm{T}$ et al (2020) Early postmortem brain MRI findings in COVID19 non-survivors. Neurology 95(14):e2016-e2027

Cui C et al (2021) AGTR2, one possible novel key gene for the entry of SARS-CoV-2 into human cells. IEEE/ACM Trans Comput Biol Bioinform 18(4):1230-1233

Cummings MJ et al (2020) Epidemiology, clinical course, and outcomes of critically ill adults with COVID-19 in New York City: a prospective cohort study. Lancet 395(10239): 1763-1770

Dai J et al (2019) Identification of risk loci and a polygenic risk score for lung cancer: a large-scale prospective cohort study in Chinese populations. Lancet Respir Med 7(10):881-891

Dalager-Pedersen M et al (2014) Risk for myocardial infarction and stroke after community-acquired bacteremia: a 20-year population-based cohort study. Circulation 129(13):1387-1396

Dantzer R (2018) Neuroimmune interactions: from the brain to the immune system and vice versa. Physiol Rev 98(1):477-504

Dendrou CA et al (2016) Resolving TYK2 locus genotype-tophenotype differences in autoimmunity. Sci Transl Med 8(363):363ra149 
Deng J et al (2021) The prevalence of depression, anxiety, and sleep disturbances in COVID-19 patients: a meta-analysis. Ann NY Acad Sci 1486(1):90-111

Desforges $M$ et al (2007) Activation of human monocytes after infection by human coronavirus 229E. Virus Res 130(1-2):228-240

Diao B et al (2020) Reduction and functional exhaustion of T cells in patients with Coronavirus Disease 2019 (COVID-19). Front Immunol 11:827

Diao B et al (2021) Human kidney is a target for novel severe acute respiratory syndrome coronavirus 2 infection. Nat Commun 12(1):2506

Dosch SF, Mahajan SD, Collins AR (2009) SARS coronavirus spike protein-induced innate immune response occurs via activation of the NF-kappaB pathway in human monocyte macrophages in vitro. Virus Res 142(1-2):19-27

Dube $\mathrm{M}$ et al (2018) Axonal transport enables neuron-to-neuron propagation of human Coronavirus OC43. J Virol 92(17):e00404

Duong L, Xu P, Liu A (2020) Meningoencephalitis without respiratory failure in a young female patient with COVID-19 infection in Downtown Los Angeles, early April 2020. Brain Behav Immun $87: 33$

Efe IE et al (2020) COVID-19-associated encephalitis mimicking glial tumor. World Neurosurg 140:46-48

Ellul MA et al (2020) Neurological associations of COVID-19. Lancet Neurol 19(9):767-783

Ely EW et al (2001) Delirium in mechanically ventilated patients: validity and reliability of the confusion assessment method for the intensive care unit (CAM-ICU). JAMA 286(21):2703-2710

Erickson MA, Banks WA (2018) Neuroimmune axes of the bloodbrain barriers and blood-brain interfaces: bases for physiological regulation, disease states, and pharmacological interventions. Pharmacol Rev 70(2):278-314

Espindola OM et al (2021) Inflammatory cytokine patterns associated with neurological diseases in Coronavirus Disease 2019. Ann Neurol 89(5):1041-1045

Fajgenbaum DC, June CH (2020) Cytokine Storm. N Engl J Med 383(23):2255-2273

Fan Z et al (2020) Clinical features of COVID-19-related liver functional abnormality. Clin Gastroenterol Hepatol 18(7):1561-1566

Feng P, Huang L, Wang H (2014) Taste bud homeostasis in health, disease, and aging. Chem Senses 39(1):3-16

Ferrante L et al (2020) Brazil's policies condemn Amazonia to a second wave of COVID-19. Nat Med 26(9):1315

Fingerlin TE et al (2013) Genome-wide association study identifies multiple susceptibility loci for pulmonary fibrosis. Nat Genet 45(6):613-620

Fliegauf M, Benzing T, Omran H (2007) When cilia go bad: cilia defects and ciliopathies. Nat Rev Mol Cell Biol 8(11):880-893

Fridman S et al (2020) Stroke risk, phenotypes, and death in COVID19: systematic review and newly reported cases. Neurology 95(24):e3373-e3385

Gerdes JC et al (1981) Coronavirus isolates SK and SD from multiple sclerosis patients are serologically related to murine coronaviruses A59 and JHM and human coronavirus OC43, but not to human coronavirus 229E. J Virol 38(1):231-238

Giulio Sancini MG, Salvati E, Cambianica I, Re F, Ornaghi F, Canovi M, Fracasso C, Alfredo Cagnotto MC, Zona C, Gobbi M, Salmona M, La Ferla B, Nicotra F, Masserini M (2013) Functionalization with TAT-peptide enhances blood-brain barrier crossing in vitro of nanoliposomes carrying a curcumin-derivative to bind amyloid-b peptide. J Nanomed Nanotechol 4(1000171):1-8

Goshua G et al (2020) Endotheliopathy in COVID-19-associated coagulopathy: evidence from a single-centre, cross-sectional study. Lancet Haematol 7(8): e575-e582

Goyal P et al (2020) Clinical characteristics of Covid-19 in New York City. N Engl J Med 382(24):2372-2374
Group R.C (2021) Dexamethasone in hospitalized patients with Covid19. N Engl J Med 384(8):693-704

Gutierrez-Ortiz C et al (2020) Miller Fisher syndrome and polyneuritis cranialis in COVID-19. Neurology 95(5):e601-e605

Hacisuleyman E et al (2021) Vaccine breakthrough infections with SARS-CoV-2 variants. N Engl J Med 384(23):2212-2218

Hadjadj $J$ et al (2020) Impaired type I interferon activity and inflammatory responses in severe COVID-19 patients. Science 369(6504):718-724

Hampshire A et al (2021) Cognitive deficits in people who have recovered from COVID-19. EClinicalMedicine 39:101044

Harrison AG, Lin T, Wang P (2020) Mechanisms of SARSCoV-2 transmission and pathogenesis. Trends Immunol 41(12):1100-1115

Hassanzadeh K et al (2020) Considerations around the SARS-CoV-2 spike protein with particular attention to COVID-19 brain infection and neurological symptoms. ACS Chem Neurosci 11(15):2361-2369

Helms J et al (2020a) Delirium and encephalopathy in severe COVID19: a cohort analysis of ICU patients. Crit Care 24(1):491

Helms J et al (2020b) High risk of thrombosis in patients with severe SARS-CoV-2 infection: a multicenter prospective cohort study. Intensive Care Med 46(6):1089-1098

Helms J et al (2020c) Neurologic features in severe SARS-CoV-2 infection. N Engl J Med 382(23):2268-2270

Heneka MT et al (2020) Immediate and long-term consequences of COVID-19 infections for the development of neurological disease. Alzheimers Res Ther 12(1):69

Hernández VS, Zetter MA, Guerra EC, Hernández-Araiza I, Karuzin N, Hernández-Pérez OR, Eiden LE, Zhang L (2021) ACE2 expression in rat brain: implications for COVID-19 associated neurological manifestations. Exp Neurol 345:113837. https://doi. org/10.1101/2021.05.01.442293

Hoelscher C et al (2020) Cerebral deep venous thrombosis and COVID19: case report. J Neurosurg 135:1-4

Hoffmann M et al (2020) SARS-CoV-2 cell entry depends on ACE2 and TMPRSS 2 and is blocked by a clinically proven protease inhibitor. Cell 181(2):271-280e8

Holmes D (2017) Repairing the neural highway. Nature 552(7684):S50-S51

Hovnanian A et al (1998) The human 2',5'-oligoadenylate synthetase locus is composed of three distinct genes clustered on chromosome 12q24.2 encoding the 100-, 69-, and 40-kDa forms. Genomics 52(3):267-277

Huang I, Pranata R (2020) Lymphopenia in severe coronavirus disease-2019 (COVID-19): systematic review and meta-analysis. J Intensive Care 8:36

Huang J et al (2020a) Potential of SARS-CoV-2 to cause CNS infection: biologic fundamental and clinical experience. Front Neurol 11:659

Huang YH, Jiang D, Huang JT (2020b) SARS-CoV-2 detected in cerebrospinal fluid by PCR in a case of COVID-19 encephalitis. Brain Behav Immun 87:149

Hughes $S$ et al (2020) Bacterial and fungal coinfection among hospitalized patients with COVID-19: a retrospective cohort study in a UK secondary-care setting. Clin Microbiol Infect 26(10):1395-1399

Iadecola C, Anrather J, Kamel H (2020) Effects of COVID-19 on the nervous system. Cell 183(1):16-27e1

Inciardi RM et al (2020) Characteristics and outcomes of patients hospitalized for COVID-19 and cardiac disease in Northern Italy. Eur Heart J 41(19): 1821-1829

Initiative C-HG (2021) Mapping the human genetic architecture of COVID-19. Nature

James N, Menzies M, Radchenko P (2021) COVID-19 second wave mortality in Europe and the United States. Chaos 31(3):031105 
Jaywant A et al (2021) Frequency and profile of objective cognitive deficits in hospitalized patients recovering from COVID-19. Neuropsychopharmacol. https://doi.org/10.1038/ s41386-021-00978-8

Kantonen J et al (2020) Neuropathologic features of four autopsied COVID-19 patients. Brain Pathol 30(6):1012-1016

Kennedy M et al (2020) Delirium in older patients with COVID-19 presenting to the emergency department. JAMA Netw Open 3(11):e2029540

Klein DE et al (2020) Cerebral venous thrombosis: a typical presentation of COVID-19 in the young. J Stroke Cerebrovasc Dis 29(8): 104989

Klok FA et al (2020) Incidence of thrombotic complications in critically ill ICU patients with COVID-19. Thromb Res 191:145-147

Kolilekas L et al (2020) Can steroids reverse the severe COVID-19 induced "cytokine storm"? J Med Virol 92(11):2866-2869

Kopanska $\mathrm{M}$ et al (2021) Changes in EEG recordings in COVID-19 patients as a basis for more accurate QEEG diagnostics and EEG neurofeedback therapy: a systematic review. J Clin Med 10(6): 1300

Koyuncu OO, Hogue IB, Enquist LW (2013) Virus infections in the nervous system. Cell Host Microbe 13(4):379-393

Lazartigues E, Qadir MMF, Mauvais-Jarvis F (2020) Endocrine significance of SARS-CoV-2's reliance on ACE2. Endocrinology 161(9):bqaa108

Lee JS, Shin EC (2020) The type I interferon response in COVID-19: implications for treatment. Nat Rev Immunol 20(10):585-586

Lee SG, Fralick M, Sholzberg M (2020) Coagulopathy associated with COVID-19. CMAJ 192(21):E583

Lei $\mathrm{F}$ et al (2020) Longitudinal association between markers of liver injury and mortality in COVID-19 in China. Hepatology 72(2):389-398

Li Y, Xiao SY (2020) Hepatic involvement in COVID-19 patients: pathology, pathogenesis, and clinical implications. J Med Virol 92(9):1491-1494

Li MY et al (2020a) Expression of the SARS-CoV-2 cell receptor gene ACE2 in a wide variety of human tissues. Infect Dis Poverty 9(1):45

Li YC, Bai WZ, Hashikawa T (2020b) The neuroinvasive potential of SARS-CoV2 may play a role in the respiratory failure of COVID-19 patients. J Med Virol 92(6):552-555

Li Y et al (2020c) Acute cerebrovascular disease following COVID19: a single center, retrospective, observational study. Stroke Vasc Neurol 5(3):279-284

Liu J et al (2020) Longitudinal characteristics of lymphocyte responses and cytokine profiles in the peripheral blood of SARS-CoV-2 infected patients. EBioMedicine 55:102763

Lodigiani C et al (2020) Venous and arterial thromboembolic complications in COVID-19 patients admitted to an academic hospital in Milan, Italy. Thromb Res 191:9-14

Lopes-Pacheco M et al (2021) Pathogenesis of multiple organ injury in COVID-19 and potential therapeutic strategies. Front Physiol 12:593223

Lu S et al (2020) First report of manic-like symptoms in a COVID19 patient with no previous history of a psychiatric disorder. J Affect Disord 277:337-340

Luan G et al (2016) Upregulation of HMGB1, toll-like receptor and RAGE in human Rasmussen's encephalitis. Epilepsy Res 123:36-49

Lutfalla G et al (1995) Mutant U5A cells are complemented by an interferon-alpha beta receptor subunit generated by alternative processing of a new member of a cytokine receptor gene cluster. EMBO J 14(20):5100-5108

Maas MB (2020) Critical medical illness and the nervous system. Continuum (minneap Minn) 26(3):675-694
Magro C et al (2020) Complement associated microvascular injury and thrombosis in the pathogenesis of severe COVID-19 infection: a report of five cases. Transl Res 220:1-13

Mallapaty S (2021) India's massive COVID surge puzzles scientists. Nature 592(7856):667-668

Mao L et al (2020) Neurologic manifestations of hospitalized patients with Coronavirus Disease 2019 in Wuhan, China. JAMA Neurol 77(6):683-690

Marjot T et al (2021) COVID-19 and liver disease: mechanistic and clinical perspectives. Nat Rev Gastroenterol Hepatol 18(5):348-364

Masuo $\mathrm{Y}$ et al (2021) Smell and stress response in the brain: review of the connection between chemistry and neuropharmacology. Molecules 26(9):2571

Matloubian M et al (2000) A transmembrane CXC chemokine is a ligand for HIV-coreceptor Bonzo. Nat Immunol 1(4):298-304

Maury A et al (2021) Neurological manifestations associated with SARS-CoV-2 and other coronaviruses: a narrative review for clinicians. Rev Neurol (paris) 177(1-2):51-64

Mawhinney JA et al (2020) Neurotropism of SARS-CoV-2: COVID19 presenting with an acute manic episode. BMJ Case Rep 13(6):e236123

McAbee GN et al (2020) Encephalitis associated with COVID-19 infection in an 11-year-old child. Pediatr Neurol 109:94

Medeiros AK et al (2020) Higher frequency of hepatic steatosis at CT among COVID-19-positive patients. Abdom Radiol (NY) 45(9):2748-2754

Mehta P et al (2020) COVID-19: consider cytokine storm syndromes and immunosuppression. Lancet 395(10229):1033-1034

Meinhardt J et al (2021) Olfactory transmucosal SARS-CoV-2 invasion as a port of central nervous system entry in individuals with COVID-19. Nat Neurosci 24(2):168-175

Merad M, Martin JC (2020) Pathological inflammation in patients with COVID-19: a key role for monocytes and macrophages. Nat Rev Immunol 20(6):355-362

Merkler AE et al (2020) Risk of ischemic stroke in patients with Coronavirus Disease 2019 (COVID-19) vs patients with influenza. JAMA Neurol 78:497

Middeldorp S et al (2020) Incidence of venous thromboembolism in hospitalized patients with COVID-19. J Thromb Haemost 18(8):1995-2002

Middleton EA et al (2020) Neutrophil extracellular traps contribute to immunothrombosis in COVID-19 acute respiratory distress syndrome. Blood 136(10):1169-1179

Mokhtari T et al (2020) COVID-19 and multiorgan failure: a narrative review on potential mechanisms. J Mol Histol 51(6):613-628

Moore JB, June CH (2020) Cytokine release syndrome in severe COVID-19. Science 368(6490):473-474

Moores LK et al (2020) Prevention, diagnosis, and treatment of VTE in patients with Coronavirus Disease 2019: CHEST guideline and expert panel report. Chest 158(3):1143-1163

Moriguchi T et al (2020) A first case of meningitis/encephalitis associated with SARS-Coronavirus-2. Int J Infect Dis 94:55-58

Moutal A et al (2021) SARS-CoV-2 spike protein co-opts VEGF$\mathrm{A} /$ neuropilin-1 receptor signaling to induce analgesia. Pain 162(1):243-252

Ou X et al (2020) Characterization of spike glycoprotein of SARSCoV-2 on virus entry and its immune cross-reactivity with SARS-CoV. Nat Commun 11(1):1620

Oxley TJ et al (2020) Large-vessel stroke as a presenting feature of Covid-19 in the young. N Engl J Med 382(20):e60

Pang HB et al (2014) An endocytosis pathway initiated through neuropilin- 1 and regulated by nutrient availability. Nat Commun 5:4904 
Paniz-Mondolfi A et al (2020) Central nervous system involvement by severe acute respiratory syndrome coronavirus-2 (SARS-CoV-2). J Med Virol 92(7):699-702

Pendlebury ST, Rothwell PM, Oxford S (2019) Vascular, Incidence and prevalence of dementia associated with transient ischaemic attack and stroke: analysis of the population-based Oxford Vascular Study. Lancet Neurol 18(3):248-258

Perico L et al (2021) Immunity, endothelial injury and complement-induced coagulopathy in COVID-19. Nat Rev Nephrol 17(1):46-64

Plein A, Fantin A, Ruhrberg C (2014) Neuropilin regulation of angiogenesis, arteriogenesis, and vascular permeability. Microcirculation 21(4):315-323

Politi LS, Salsano E, Grimaldi M (2020) Magnetic resonance imaging alteration of the brain in a patient with Coronavirus Disease 2019 (COVID-19) and Anosmia. JAMA Neurol 77(8):1028-1029

Qi F et al (2020) Single cell RNA sequencing of 13 human tissues identify cell types and receptors of human coronaviruses. Biochem Biophys Res Commun 526(1):135-140

Qin C et al (2020) Dysregulation of immune response in patients with Coronavirus 2019 (COVID-19) in Wuhan, China. Clin Infect Dis 71(15):762-768

Ramlall V et al (2020) Immune complement and coagulation dysfunction in adverse outcomes of SARS-CoV-2 infection. Nat Med 26(10):1609-1615

Reichard RR et al (2020) Neuropathology of COVID-19: a spectrum of vascular and acute disseminated encephalomyelitis (ADEM)-like pathology. Acta Neuropathol 140(1):1-6

Reiter ER et al (2020) Subjective smell and taste changes during the COVID-19 pandemic: Short term recovery. Am J Otolaryngol 41(6): 102639

Rossaint J et al (2014) Synchronized integrin engagement and chemokine activation is crucial in neutrophil extracellular trapmediated sterile inflammation. Blood 123(16):2573-2584

Rostami M, Mansouritorghabeh H (2020) D-dimer level in COVID19 infection: a systematic review. Expert Rev Hematol 13(11):1265-1275

Schultze JL, Aschenbrenner AC (2021) COVID-19 and the human innate immune system. Cell 184(7):1671-1692

Seo $S$ et al (2011) A novel protein LZTFL1 regulates ciliary trafficking of the BBSome and Smoothened. PLoS Genet 7(11):e1002358

Shang J et al (2020) Cell entry mechanisms of SARS-CoV-2. Proc Natl Acad Sci USA 117(21):11727-11734

Shen B et al (2020) Proteomic and metabolomic characterization of COVID-19 patient sera. Cell 182(1):59-72e15

Shu $\mathrm{T}$ et al (2020) Plasma proteomics identify biomarkers and pathogenesis of COVID-19. Immunity 53(5):1108-1122e5

Smith EM et al (1999) IL-10 as a mediator in the HPA axis and brain. J Neuroimmunol 100(1-2):140-148

Smith ER et al (2005) A human protein complex homologous to the Drosophila MSL complex is responsible for the majority of histone $\mathrm{H} 4$ acetylation at lysine 16. Mol Cell Biol 25(21):9175-9188

Solomon IH et al (2020) Neuropathological features of Covid-19. N Engl J Med 383(10):989-992

Song E et al (2021) Neuroinvasion of SARS-CoV-2 in human and mouse brain. J Exp Med 218(3):e20202135

Spence JD et al (2020) Mechanisms of stroke in COVID-19. Cerebrovasc Dis 49(4):451-458

$\mathrm{Su} \mathrm{H}$ et al (2020) Renal histopathological analysis of 26 postmortem findings of patients with COVID-19 in China. Kidney Int 98(1):219-227

Suh YJ et al (2021) Pulmonary embolism and deep vein thrombosis in COVID-19: a systematic review and meta-analysis. Radiology 298(2):E70-E80
Sultan-Styne K et al (2009) Long-term survival of olfactory sensory neurons after target depletion. J Comp Neurol 515(6):696-710

Tilley AE et al (2015) Cilia dysfunction in lung disease. Annu Rev Physiol 77:379-406

Toscano G et al (2020) Guillain-Barre syndrome associated with SARS-CoV-2. N Engl J Med 382(26):2574-2576

Tummino TA et al (2021) Drug-induced phospholipidosis confounds drug repurposing for SARS-CoV-2. Science 373(6554):541-547

Underwood MD et al (1999) Morphometry of the dorsal raphe nucleus serotonergic neurons in suicide victims. Biol Psychiatry 46(4):473-483

Vankadari N, Wilce JA (2020) Emerging WuHan (COVID-19) coronavirus: glycan shield and structure prediction of spike glycoprotein and its interaction with human CD26. Emerg Microbes Infect 9(1):601-604

Venkatesan A et al (2013) Case definitions, diagnostic algorithms, and priorities in encephalitis: consensus statement of the international encephalitis consortium. Clin Infect Dis 57(8):1114-1128

Venketasubramanian N, Hennerici MG (2020) Stroke in COVID-19 and SARS-CoV-1. Cerebrovasc Dis 49(3):235-236

Virhammar J et al (2020) Acute necrotizing encephalopathy with SARS-CoV-2 RNA confirmed in cerebrospinal fluid. Neurology 95(10):445-449

von Hundelshausen P et al (2005) Heterophilic interactions of platelet factor 4 and RANTES promote monocyte arrest on endothelium. Blood 105(3):924-930

Wan Y et al (2020) Receptor recognition by the Novel Coronavirus from Wuhan: an analysis based on decade-long structural studies of SARS Coronavirus. J Virol 94(7):e00127

Wang K et al (2020a) CD147-spike protein is a novel route for SARSCoV-2 infection to host cells. Signal Transduct Target Ther $5(1): 283$

Wang L et al (2020b) Prolonged prothrombin time at admission predicts poor clinical outcome in COVID-19 patients. World J Clin Cases 8(19):4370-4379

Wang D et al (2020c) Clinical characteristics of 138 hospitalized patients with 2019 novel coronavirus-infected pneumonia in Wuhan, China. JAMA 323(11):1061-1069

Wang L et al (2021) An atlas connecting shared genetic architecture of human diseases and molecular phenotypes provides insight into COVID-19 susceptibility. Genome Med 13(1):83

WHO (2021) WHO Coronavirus (COVID-19) Dashboard

Wilkerson RG et al (2020) Silent hypoxia: a harbinger of clinical deterioration in patients with COVID-19. Am J Emerg Med 38(10):2243e5-2243e6

$\mathrm{Xu} \mathrm{H}$ et al (2020) Clinical characteristics and risk factors of cardiac involvement in COVID-19. J Am Heart Assoc 9(18):e016807

$\mathrm{Xu} \mathrm{Z}$ et al (2020) Pathological findings of COVID-19 associated with acute respiratory distress syndrome. Lancet Respir Med 8(4):420-422

Yaghi S et al (2020) SARS-CoV-2 and stroke in a new york healthcare system. Stroke 51(7):2002-2011

Yang L et al (2020) A Human pluripotent stem cell-based platform to study SARS-CoV-2 tropism and model virus infection in human cells and organoids. Cell Stem Cell 27(1):125-136

Ye M, Ren Y, Lv T (2020) Encephalitis as a clinical manifestation of COVID-19. Brain Behav Immun 88:945-946

Yeager CL et al (1992) Human aminopeptidase $\mathrm{N}$ is a receptor for human coronavirus 229E. Nature 357(6377):420-422

Zanin L et al (2020) SARS-CoV-2 can induce brain and spine demyelinating lesions. Acta Neurochir (wien) 162(7):1491-1494

Zeqing Feng BD, Rongshuai W, Gang W, Chenhui W, Yingjun T, Liang L, Changsong W, Ying L, Yueping L, Zilin Y, Liang R, Yuzhang $\mathrm{W}$, Yongwen C (2020) The novel severe acute respiratory syndrome Coronavirus 2 (SARS-CoV-2) directly decimates human 
spleens and lymph nodes. https://doi.org/10.1101/2020.03.27. 20045427

Zhang T et al (2016) CaMKII is a RIP3 substrate mediating ischemiaand oxidative stress-induced myocardial necroptosis. Nat Med 22(2):175-182

Zhang C et al (2020) Intracellular autoactivation of TMPRSS11A, an airway epithelial transmembrane serine protease. J Biol Chem 295(36):12686-12696

Zhen L, Jiwei YMW, Jie G, Xiang L, Siji S, Jiali L, Guangjie D, Yuanxiu Z, Xiaojun W, Zhansong Z, Taojiao W, Ming H, Xianxiang C, Yu F, Chong L, Hailong D, Chuou X, Yahua H, Min H, Yi Z, Hongbo J, Xiaowei C, Junan Y (2020) Caution on Kidney Dysfunctions of COVID-19 Patients. https://doi.org/10.1101/ 2020.02.08.20021212

Zheng S et al (2020a) Viral load dynamics and disease severity in patients infected with SARS-CoV-2 in Zhejiang province, China, January-March 2020: retrospective cohort study. BMJ 369:m1443

Zheng YY et al (2020b) COVID-19 and the cardiovascular system. Nat Rev Cardiol 17(5):259-260
Zhou W, Wang Y (2016) Candidate genes of idiopathic pulmonary fibrosis: current evidence and research. Appl Clin Genet 9:5-13

Zhou P et al (2020a) A pneumonia outbreak associated with a new coronavirus of probable bat origin. Nature 579(7798):270-273

Zhou Y et al (2020b) Network-based drug repurposing for novel coronavirus 2019-nCoV/SARS-CoV-2. Cell Discov 6(1):14

Zingg B et al (2020) Synaptic specificity and application of anterograde transsynaptic AAV for probing neural circuitry. J Neurosci 40(16):3250-3267

Zou X et al (2020) Single-cell RNA-seq data analysis on the receptor ACE2 expression reveals the potential risk of different human organs vulnerable to 2019-nCoV infection. Front Med 14(2):185-192

Publisher's Note Springer Nature remains neutral with regard to jurisdictional claims in published maps and institutional affiliations. 